\title{
Peripheral Intraneural Angiosarcoma
}

National Cancer Institute

\section{Source}

National Cancer Institute. Peripheral Intraneural Angiosarcoma. NCI Thesaurus. Code C5320.

A malignant vascular neoplasm arising from the peripheral nerves. 